\title{
Effectiveness of Granisetron in Prevention of Hypotension Following Spinal Anaesthesia in Patients Undergoing Elective Caesarean Section
}

Abhishek Chatterjee ${ }^{1}$, Bhanu Gudiwada ${ }^{2}$, Pratap Rudra Mahanty ${ }^{1}$, Himanshu Kumar ${ }^{1}$, Deb Sanjay Nag ${ }^{1}$, Pradip Kumar Ganguly ${ }^{1}$, Rajiv Shukla ${ }^{1}$

1. Anaesthesiology, Tata Main Hospital, Jamshedpur, IND 2. Anaesthesiology, Varma Hospital, Bhimavaram, IND

Corresponding author: Abhishek Chatterjee,dr.abhishekchatterjee@gmail.com
Review began $11 / 30 / 2020$ Review ended 12/08/2020 Published 12/16/2020

\section{() Copyright 2020}

Chatterjee et al. This is an open access article distributed under the terms of the Creative Commons Attribution License CC-BY 4.0., which permits unrestricted use, distribution, and reproduction in any medium, provided the original author and source are credited.

\begin{abstract}
Background and aim: Spinal anesthesia is the most common type of anesthesia administered for caesarean section and it is frequently associated with hypotension. When post-spinal hypotension is accompanied with bradycardia, the condition may become more complicated. Numerous pharmacological agents have therefore been tried for prevention of hypotension and 5HT3 antagonists are the latest in the armamentarium. However, studies have shown conflicting evidence regardings the effectiveness of 5HT3 inhibitors (ondansetron and granisetron) in preventing spinal hypotension. We have tried to address this controversy and also wanted to explore the adverse effects of granisetron on the foetus, if any.
\end{abstract}

Materials and methods: Two hundred patients were included in the study and divided into two groups of 100 patients each. Group S patients received $5 \mathrm{ml}$ of $0.9 \%$ normal saline while Group G patients received IV granisetron $1 \mathrm{mg}$ (diluted to $5 \mathrm{ml}$ ) 10 minutes prior to administration of spinal anesthesia. Analysis of variance (ANOVA) test was used for comparing the data, Student t-test was applied to compare the difference between the two means and Chi-Square test was used to test significance of difference of proportions.

Results: The incidence of hypotension in Group S was 69\%, whereas it was 37\% in Group G (p<0.001), hence patients of Group $S$ required a significantly higher $(\mathrm{p}=0.001)$ amount of mephentermine. Haemodynamic parameters were well maintained throughout the study period in patients of Group G. The neonatal outcome was assessed by Apgar score at 0 minutes, one minute, and five minutes after delivery, and it was comparable between the two study groups.

Conclusion: Intravenous granisetron $1 \mathrm{mg}$ if administered before administering spinal anesthesia can effectively attenuate hypotension in parturients without any adverse effects on the mother and the neonate.

Categories: Anesthesiology, Obstetrics/Gynecology

Keywords: granisetron, hypotension, spinal anesthesia, caesarean section

\section{Introduction}

The most commonly administered anaesthesia technique for conducting caesarean section is subarachnoid block (SAB). SAB, although considered safe, has a reported incidence of hypotension following the procedure as high as $20-40 \%$ in pregnant patients [1]. Similarly, bradycardia is also commonly associated with post-SAB and reported incidence is around 13\% [2]. Spinal anaesthesia results in sympathetic block leading to a decrease in systemic vascular resistance and hypotension, which can be minimised or prevented by various methods like left uterine displacement [3], preloading [4], or co-loading [5] with crystalloids [4] or colloids [6], use of vasopressors like ephedrine [7], mephentermine [8], phenylephrine [9], etc. Hypotension caused by $\mathrm{SAB}$ is physiologically compensated by an increase in heart rate. However, if vagus nerve-mediated cardiodepressor reflex-like Bezold-Jarisch reflex (BJR) gets stimulated, then the cardiac autonomic balance gets shifted towards the parasympathetic nervous system leading to bradycardia, which further precipitates hypotension [10]. The combination of hypotension with bradycardia has been a matter of concern for all anaesthesiologists and therefore, using an effective agent to take care of BJR is of prime importance in management of hypotension following SAB. Both mechanoreceptors as well as chemoreceptors are responsible for hypotension and bradycardia after spinal blockade. Deformation of cardiac walls following hypovolemia after SAB stimulates the mechanoreceptors present in cardiac chambers which further leads to initiation of Bezold-Jarisch reflex. The chemoreceptors are activated by serotonin released from activated thrombocytes [11,12].

Owczuk et al. [13] and Sahoo et al. [14] conducted studies in human beings with ondansetron for attenuation of post-spinal hypotension by inhibiting BJR and they found it to be effective. Similarly, studies carried out with granisetron by Khalifa [15], Edalba et al. [16], Mohammadi et al. [17], and Megahed et al. [18] found that it was effective in prevention of hypotension following SAB. However, contrary to the above findings, 
Shrestha et al. [19], Lamichhane et al. [20], and Saberi et al. [21] found granisetron was not effective in prevention of hypotension following SAB.

Therefore, this study was undertaken with the aim of addressing the ongoing controversy regarding effectiveness of granisetron in preventing hypotension following SAB in obstetric patients undergoing caesarean section. The primary objective was to assess the effectiveness of $1 \mathrm{mg}$ intravenous granisetron in prevention of hypotension and the secondary objective was to assess the effect of granisetron in preventing bradycardia following spinal anaesthesia in parturients undergoing lower segment caesarean section (LSCS).

\section{Materials And Methods}

This was a prospective double-blind randomized controlled trial. A total of 200 healthy term parturients (between 20 - 35 years) undergoing elective caesarean section under spinal anaesthesia were included in the study. They were then divided into two groups with 100 patients in each group (Precision 0.05, Power of study - 80\%, Incidence of hypotension after SAB - 20\%). Parturients not willing to participate in this study, presence of any contraindication for spinal anaesthesia, history of allergy to study drug, patients on serotonin agonists or antagonists, and presence of any co-existing diseases were excluded from the study.

Parturients were randomized into one of the two groups using a computer-based random number generator. The study drugs were premixed to a volume of $5 \mathrm{ml}$ and presented as coded syringes to the anaesthesiologist who was not an investigator in the study. Group $\mathrm{G}$ patients received IV granisetron $1 \mathrm{mg}$ and Group S patients received IV $5 \mathrm{ml}$ of $0.9 \%$ normal saline. Both the groups received the allocated solution intravenously 10 minutes before spinal anaesthesia. All parameters were noted by an anaesthesiologist blinded to the group allocation. All parturients were kept fasting for eight hours and they were uniformly pre-medicated with ranitidine $150 \mathrm{mg}$ and metoclopramide $10 \mathrm{mg}$ orally on the morning of surgery with sips of water.

Baseline/pre-spinal (T0) vital parameters of parturients including heart rate (HR), systolic blood pressure (SBP), diastolic blood pressure (DBP), mean arterial blood pressure (MAP), and peripheral oxygen saturation (SpO2) were recorded in the operating room. Any fall in the systolic arterial blood pressure below $100 \mathrm{mmHg}$ or a fall in mean arterial blood pressure of more than $20 \%$ from baseline value was taken as hypotension and managed with intravenous mephentermine $6 \mathrm{mg}$ bolus. In the operating room, an IV line was secured with $18-\mathrm{G}$ intravenous cannula and infusion of ringer lactate solution started. Routine standard monitors such as pulse oximetry, electrocardiography (ECG) and non-invasive blood pressure applied and monitored. Subarachnoid block was given in a sitting position with midline approach with a disposable 27 gauge Quincke spinal needle. After confirming the free flow of cerebrospinal fluid, $2.2 \mathrm{ml}$ of $0.5 \%$ hyperbaric bupivacaine was deposited in the subarachnoid space at the rate of $1 \mathrm{ml}$ over 10 seconds. Parturients were made supine after putting a sterile dressing over the skin at the lumbar puncture site. The time of intra-thecal drug deposition noted and the haemodynamic parameters were recorded. All the above-mentioned parameters were recorded at every two-minute interval till 20 minutes after SAB (T-1 T-2 T-3 and so on up to T-10) and then at every five minutes till the end of surgery (T-11 onwards) [17]. All the haemodynamic parameters were monitored again in the postoperative period (T-17) after one hour of surgery [22]. Further monitoring of all patients of both the study groups were continued as per institutional protocol in the recovery area. Furthermore, other parameters were also recorded like height of sensory block (by pin-prick at midclavicular line with blunt needle from below upwards) after five minutes of SAB, duration of surgery, amount of intra operative blood loss, total requirement of IV mephentermine, need of IV atropine to treat bradycardia, and Apgar score (Appearance, Pulse, Grimace, Activity, and Respiration) of the neonate (at birth, at one minute, and at five minutes).

Analysis of variance (ANOVA) was used for comparing the data, Student t-test was applied to compare the difference between two means and Chi-Square test was used to test significance of difference of proportions.

\section{Results}

The patient characteristics like age, weight, height, body mass index were comparable in both the groups. The baseline haemodynamic parameters like the heart rate, systolic blood pressure, diastolic blood pressure, mean arterial blood pressure, and peripheral oxygen saturation was comparable in both the groups before giving spinal anesthesia.

The incidence of hypotension in Group S (69\%) was more than Group G (37\%) (Table 1) and the difference between the two groups was statistically significant $(\mathrm{p}<0.001)$ 


\section{Cureus}

\begin{tabular}{|c|c|c|c|c|c|}
\hline \multirow{2}{*}{ Hypotension } & \multicolumn{2}{|l|}{ Group S } & \multicolumn{2}{|l|}{ Group G } & \multirow{2}{*}{$p$ value } \\
\hline & Frequency & $\%$ & Frequency & $\%$ & \\
\hline No & 31 & $31.0 \%$ & 63 & $63.0 \%$ & \multirow{3}{*}{$<0.001$} \\
\hline Yes & 69 & $69.0 \%$ & 37 & $37.0 \%$ & \\
\hline Total & 100 & $100 \%$ & 100 & $100 \%$ & \\
\hline
\end{tabular}

\section{TABLE 1: Incidence of hypotension}

Use of mephentermine as vasopressor was also significantly higher in Group S as compared to Group G. None of the patients required atropine (Table 2).

\begin{tabular}{|c|c|c|c|}
\hline & Group S & Group G & \multirow{2}{*}{ p value } \\
\hline & Mean \pm SD & Mean \pm SD & \\
\hline Total Mephentermine (mg/patient) & $12.27 \pm 6.64$ & $7.82 \pm 2.80$ & $<0.001$ \\
\hline Total Atropine & Not required & Not required & - \\
\hline
\end{tabular}

TABLE 2: Use of mephentermine and atropine

On comparison of the trend of systolic blood pressure between the two study groups at different time points, the difference was significant at $\mathrm{T} 3$ (two minutes after $\mathrm{SAB}$ ), T4 (eight minutes after SAB), T6 (12 minutes after $\mathrm{SAB}$ ), T10 (20 minutes after $\mathrm{SAB}$ ) and T12 (30 minutes after SAB); p-values at these time points are $<$ 0.05 (Figure 1).

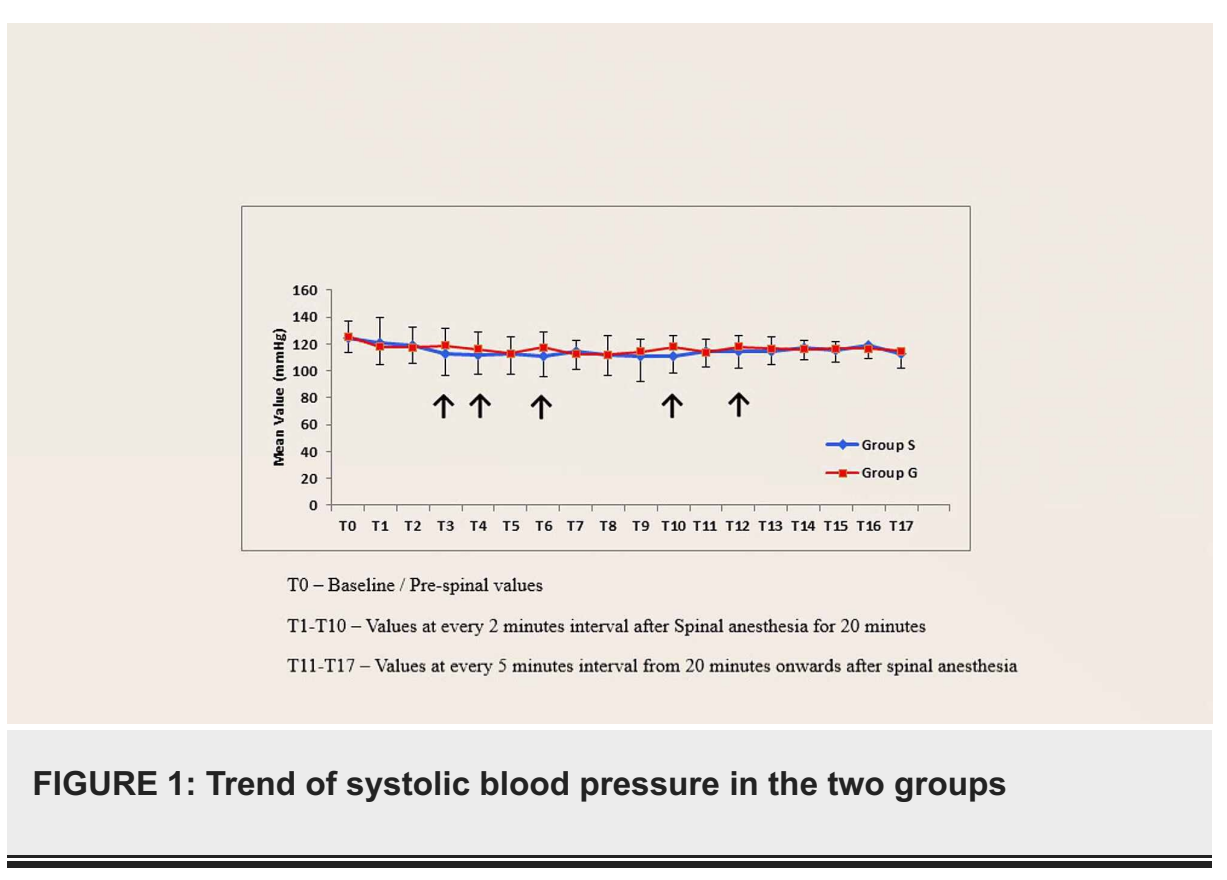

The trend of diastolic blood pressure showed that except at T12 (30 minutes after SAB), the difference between the two groups was non-significant and DBP was better maintained in the granisetron group (Figure 2). 


\section{Cureus}

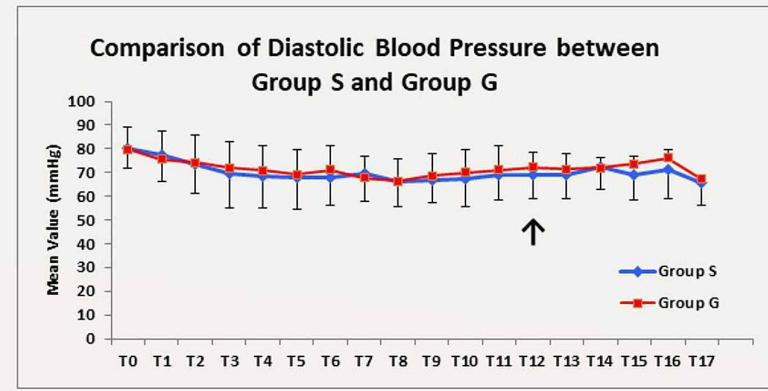

T0 - Baseline / Pre-spinal values

T1-T10 - Values at every 2 minutes interval after Spinal anesthesia for 20 minutes

T11-T17 - Values at every 5 minutes interval from 20 minutes onwards after spinal anesthesia

FIGURE 2: Trend of diastolic blood pressure in the two groups

The trend of mean arterial blood pressure (MAP) showed that at T4 (eight minutes after SAB), T6 (12 minutes after $\mathrm{SAB}$ ), T10 (20 minutes after SAB) and T12 (30 minutes after $\mathrm{SAB}$ ), the difference in MAP between two groups was significant. ( $\mathrm{p}$-values are $<0.05$ ). The mean arterial blood pressure after spinal anaesthesia was also significantly better maintained in Group $\mathrm{G}$ at different time points in comparison to Group S (Figure 3).

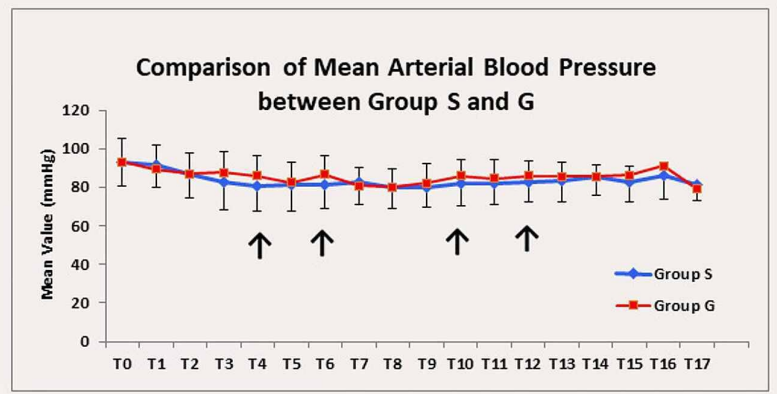

T0 - Baseline / Pre-spinal values

T1-T10 - Values at every 2 minutes interval after Spinal anesthesia for 20 minutes

T11-T17 - Values at every 5 minutes interval from 20 minutes onwards after spinal anesthesia

\section{FIGURE 3: Trend of mean arterial blood pressure in two groups}

There was no statistically significant difference between the study groups in heart rate after $\mathrm{SAB}$ ( $\mathrm{p}$-value > $0.05) /$

There was no statistically significant difference $(\mathrm{p}=0.101)$ in height of sensory block in parturients after $\mathrm{SAB}$, i.e. parturients of both groups attained comparable height of sensory block. The surgical duration and amount of intraoperative blood loss between Group S and Group $\mathrm{G}$ were also comparable ( $\mathrm{p}$-values 0.740 and 0.627 respectively).

Neonatal outcome depends upon Apgar score, which was also comparable between the two study groups at 0 minutes, one minute, and five minutes after delivery.

\section{Discussion}

Neuraxial anaesthesia remains the preferred choice for LSCS across the world and hypotension after SAB 
remains one of the most researched subjects in obstetric anaesthesia. Hypotension is the physiological consequence of spinal anaesthesia and can have potential deleterious maternal and foetal impact. Several methods are therefore being followed to treat hypotension after SAB like IV fluids, pharmacological agents, and physical methods like positioning. Over the last few years, different pharmacological agents are in use to treat post-spinal hypotension. Prevention of spinal hypotension in parturients where two lives are affected is a real concern for the anaesthesiologist and an effective and safe prophylactic pharmacological agent will be ideal for this purpose. With the introduction of 5-HT3 receptor antagonist in clinical practice, different authors started exploring these agents and since then the treatment options for spinal induced hypotension has been widened. Numerous articles have been published showing the efficacy of both ondansetron and granisetron for the purpose of management of post-spinal hypotension. However, a few studies have shown that granisetron is ineffective for this very purpose. We therefore tried to address this issue and have conducted a prospective, randomized, double-blind study with the aim to study the effectiveness of intravenous granisetron $(1 \mathrm{mg})$ in prevention of hypotension in parturients undergoing elective caesarean section under spinal anaesthesia.

In our study we observed that $67 \%$ of parturients of Group S had hypotension after spinal anaesthesia whereas only $33 \%$ of parturients developed hypotension in Group G and this difference was statistically significant with a p-value $<0.001$ (Table 1 ).

Eldaba et al. [16] in a similar study also observed a statistically significant difference with $3 \%$ incidence of hypotension in the granisetron group as compared to $64 \%$ in the normal saline group. Megahed et al. [18] and Sayed et al. [23] also observed significantly less incidence of hypotension when patients were given granisetron or ondansetron as compared to normal saline.

However, Lamichhane et al. [20] who used a similar dosage of granisetron and Shrestha et al. [19] using granisetron at $40 \mathrm{mcg} / \mathrm{kg}$ did not observe a satisfactory effect of IV granisetron. Both the authors did not take intraoperative blood loss and height of sensory block into account and perhaps the patients of the granisetron group had higher sensory block or more surgical blood loss as compared to the normal saline group. In the present study, surgical blood loss, duration of surgery, and height of sensory block were similar in both groups.

In the present study mean dose of intravenous mephentermine used for the treatment of hypotension following spinal anaesthesia was significantly low in Group G compared to Group S (Table 2). Lamichhane et al. [20] and Eldaba et al. [16] had used a similar dose of granisetron in their studies. They found reduced requirement of rescue vasopressor in the granisetron group for treatment of spinal anaesthesia induced hypotension in comparison to the normal saline group. However, Mohammadi et al. [17], although they used a higher dose of granisetron ( $3 \mathrm{mg}$ ) in their study, found a non-significant difference between the two study groups in relation to the need for vasopressor for management of post-spinal hypotension. Mohammadi et al. [17] did not mention factors like level of block and intravenous fluids used, which could have influenced the hypotensive episodes in the perioperative period.

Baseline systolic blood pressure (T-0) between the two study groups was comparable in the present study. After administering spinal anesthesia, the systolic blood pressure at different points of time was comparable between the two groups except for readings at the sixth minute, eighth minute, 12 th minute, 20th minute, and 30th minute. Different authors studied the onset and duration of post-spinal hypotension and they observed incidence of hypotension after three minutes of SAB [24] and this hypotension persisted during the intraoperative period [25]. On comparing both the above findings, the present study showed fall in SBP was greater in the saline group until 30 minutes after $\mathrm{SAB}$ and granisetron had a beneficial effect against fall in SBP after SAB. Saberi et al. [21] observed that although the granisetron group showed a lesser decrease in systolic blood pressure as compared to normal saline, the difference was not statistically significant. Shrestha et al. [19] also observed a similar non-significant difference in systolic blood pressure between the two groups. Both studies did not take surgical blood loss or other influencing factors into account and therefore the findings were different when compared to our study. In our study the height of sensory block and intraoperative blood loss were comparable in both groups.

Diastolic blood pressure between the two groups was comparable throughout the study period except at the 30th minute, which indicated granisetron had a negligible effect on diastolic blood pressure as compared to systolic blood pressure. Both Lamichhane et al. [20] and Saberi et al. [21] observed similar results in diastolic blood pressure.

In the present study, baseline mean arterial blood pressure between Group $\mathrm{G}$ and Group $\mathrm{S}$ was comparable. After giving $\mathrm{SAB}$, the difference in mean arterial blood pressure between the two groups was significantly different at the eighth, 12th, 20th, and 30th minute, which was similar to change in systolic blood pressure between the two groups at the same time interval. Mean arterial blood pressure takes into account both systolic blood pressure and diastolic blood pressure. As diastolic blood pressure did not show significant difference between the two groups, the changes that occurred in systolic blood pressure were reflected in a similar way and we observed similar significant changes in mean arterial blood pressure. When use of mephentermine was compared we observed significant difference between the two groups at the sixth 
minute, eighth minute, 12th minute, and 20th minute. Khalifa et al. [15] found that the fall of mean arterial blood pressure with granisetron and use of ephedrine was significantly low in comparison to the saline group at five minutes after SAB. Eldaba et al. [16] also concluded that granisetron significantly reduced the decrease in mean arterial blood pressure when used as a premedication before spinal anaesthesia in caesarean section and observed a prolonged median time elapse to incidence of hypotension in the granisetron group (16 minutes) as compared to the saline group (seven minutes).

In our study the baseline heart was comparable in both groups. After SAB, heart rate trend showed nonsignificant difference between Group $\mathrm{G}$ and Group S throughout the study period. Our findings of change in heart rate were similar to Lamichhane et al. [20], Mohammadi et al. [17], and Shrestha et al. [19] in two different studies; although they used higher doses of granisetron in their studies, they failed to observe significant heart rate variations.

In our study we did not find any episode of significant bradycardia in either of the groups and therefore intravenous atropine was not needed. Lamichhane et al. [20] also did not find any episode of bradycardia in either of the groups. However, Eldaba et al. [16] found significant difference in incidence of bradycardia, i.e. more number of parturients in the saline group had bradycardia in comparison to the granisetron group and they concluded that granisetron given prior to spinal anaesthesia reduced the incidence of bradycardia, perhaps due to inhibition of Bezold Jarisch reflex [16].

Incidence of hypotension after spinal anaesthesia is as high as 50-60\% [1] in the obstetric population when compared to the non-obstetric population which is around 33\% [2]. This difference may be influenced by many factors during preoperative period. To address this issue, we also observed total duration of surgery (skin incision to closure time), intra operative blood loss, height of sensory block, technique of subarachnoid block, etc. We administered subarachnoid block as per our institutional protocol to every parturient and we maintained same degree of Trendelenburg position in all parturients enrolled in the study.

In spinal anaesthesia when sensory block level attains $\mathrm{T} 4$ or above T4 dermatomal level it increases the incidence of hypotension in the obstetric population. As block level reaches at or above T4 dermatomal level, it leads to inhibition of cardiac sympathetic system which is an influencing factor on haemodynamic parameters in spinal anaesthesia [24]. So monitoring sensory block level is an important factor after administering subarachnoid block. In the current study, sensory block level attained in both groups was comparable.

Surgical blood loss also influences haemodynamic parameters and may lead to hypotension. Intraoperative blood loss along with SAB have an additive effect and may cause profound hypotension. We, therefore, explored the effect of intraoperative blood loss on incidence of post-spinal hypotension between the two groups. In our study we observed that mean intraoperative blood loss between the two study groups was also comparable.

The Apgar score describes the condition of newborns immediately after birth and when properly applied, is a tool for standardized assessment [26]. In the current study, Apgar scores at 0 minutes, one minute and five minutes were comparable between the two groups. Eldaba et al. [16] also observed similar non-significant difference in Apgar score between two groups. We therefore can say that intravenous granisetron did not have any adverse neonatal outcome.

\section{Conclusions}

Subarachnoid block is still the most commonly performed anaesthesia technique for lower segment caesarean section. Incidence of post-spinal hypotension is high in parturients undergoing lower segment caesarean section. Intravenous granisetron $1 \mathrm{mg}$ if administered before SAB, besides being an effective antiemetic, can also be used to effectively attenuate hypotension in parturients without any adverse effects on mother and neonate.

\section{Additional Information \\ Disclosures}

Human subjects: Consent was obtained by all participants in this study. Ethical Committee, Tata Main Hospital, Jamshedpur - 831001, India issued approval TMH/FRM/QMS/ALL/16. - Approved by Institutional Ethical Committee (Testing ID CD1577833), Tata Main Hospital, Jamshedpur - 831001, India - This study was also registered with Clinical Trial Registry of India (CTRI)- Trial registry no. - CTRI/2018/07/015038. Animal subjects: All authors have confirmed that this study did not involve animal subjects or tissue. Conflicts of interest: In compliance with the ICMJE uniform disclosure form, all authors declare the following: Payment/services info: All authors have declared that no financial support was received from any organization for the submitted work. Financial relationships: All authors have declared that they have no financial relationships at present or within the previous three years with any organizations that might have an interest in the submitted work. Other relationships: All authors have declared that there are no other relationships or activities that could appear to have influenced the submitted work. 


\section{References}

1. Fakherpour A, Ghaem H, Fattahi Z, Zaree S: Maternal and anaesthesia-related risk factors and incidence of spinal anaesthesia-induced hypotension in elective caesarean section: a multinomial logistic regression. Indian J Anaesth. 2018, 62:36-46. 10.4103/ija.IJA_416_17

2. Carpenter RL, Caplan RA, Brown DL, Stephenson C, Wu R: Incidence and risk factors for side effects of spinal anesthesia. Anesthesiology. 1992, 76:906-16. 10.1097/00000542-199206000-00006

3. Milsom I, Forssman L, Biber B, Dottori O, Rydgren B, Sivertsson R: Maternal haemodynamic changes during caesarean section: a comparison of epidural and general anaesthesia. Acta Anaesthesiol Scand. 1985, 29:161-7.

4. Burns SM, Cowan CM, Wilkes RG: Prevention and management of hypotension during spinal anaesthesia for elective Caesarean section: a survey of practice. Anaesthesia. 2001, 56:794-8.

5. Gunusen I, Karaman S, Ertugrul V, Firat V: Effects of fluid preload (crystalloid or colloid) compared with crystalloid co-load plus ephedrine infusion on hypotension and neonatal outcome during spinal anaesthesia for caesarean delivery. Anaesth Intensive Care. 2010, 38:647-53. 10.1177/0310057X1003800337

6. Riley ET, Cohen SE, Rubenstein AJ, Flanagan B: Prevention of hypotension after spinal anesthesia for cesarean section: six percent hetastarch versus lactated Ringer's solution. Anesth Analg. 1995, 81:838-42. 10.1097/00000539-199510000-00031

7. Marcus MA, Vertommen JD, Van Aken H, Wouters PF: Hemodynamic effects of intravenous isoproterenol versus epinephrine in the chronic maternal-fetal sheep preparation. Anesth Analg. 1996, 82:1023-6. 10.1097/00000539-199605000-00024

8. Ramanathan S, Grant GJ: Vasopressor therapy for hypotension due to epidural anesthesia for cesarean section. Acta Anaesthesiol Scand. 1988, 32:559-65. 10.1111/j.1399-6576.1988.tb02786.x

9. Westfall TC, Westfall DP: Adrenergic agonists and antagonists. Goodman \& Gilman's: The Pharmacological Basis of Therapeutics, 12e. Shanahan JF, Naglieri C (ed): McGraw-Hill Education, New York; 2006.

10. Liu SS, McDonald SB: Current issues in spinal anesthesia. Anesthesiology. 2001, 94:888-906. 10.1097/00000542-200105000-00030

11. Martinek RM: Witnessed asystole during spinal anesthesia treated with atropine and ondansetron: a case report. Can J Anaesth. 2004, 51:226-30. 10.1007/BF03019100

12. Mao HZ, Li Z, Chapleau MW: Platelet activation in carotid sinuses triggers reflex sympathoinhibition and hypotension. Hypertension. 1996, 27:584-90. 10.1161/01.hyp.27.3.584

13. Owczuk R, Wenski W, Polak-Krzeminska A, et al.: Ondansetron given intravenously attenuates arterial blood pressure drop due to spinal anesthesia: a double-blind, placebo-controlled study. Reg Anesth Pain Med. 2008, 33:332-9.

14. Sahoo T, Sen Dasgupta C, Goswami A, Hazra A: Reduction in spinal-induced hypotension with ondansetron in parturients undergoing caesarean section: a double-blind randomised, placebo-controlled study. Int J Obstet Anest. 2012, 21:24-8. 10.1016/j.ijoa.2011.08.002

15. Khalifa OS: A comparative study of prophylactic intravenous granisetron, ondansetron, and ephedrine in attenuating hypotension and its effect on motor and sensory block in elective cesarean section under spinal anesthesia. Ain-Shams J Anaest. 2015, 8:166-172. 10.4103/1687-7934.156667

16. Eldaba AA, Amr YM: Intravenous granisetron attenuates hypotension during spinal anesthesia in cesarean delivery: a double-blind, prospective randomized controlled study. J Anaesthesiol Clin Pharmacol. 2015, 31:329-32. 10.4103/0970-9185.161667

17. Mohammadi SS, Mehrpoor S, Saliminia A: Efficacy of granisetron on attenuation of hemodynamic responses of parturients undergoing elective cesarean delivery under spinal anesthesia. Arc Anest Crit Care. 2017, 3:373-7.

18. Megahed MA, Hady AM, Sayed AE, Mohamed MA: Clinical comparative study of the effects of intravenous ondansetron and granisetron on hemodynamic changes, shivering, and motor \& sensory blockade induced by spinal anesthesia in women undergoing cesarean section. NY Sci J. 2017, 10:7-16. 10.7537/marsnys 100617.02

19. Shrestha BK, Acharya SP, Marhatta MN: Use of granisetron for prevention of hypotension and bradycardia due to spinal anesthesia: a double blind randomised control trial. JSAN. 2015, 1:36-39. 10.3126/jsan.v1i1.13587

20. Lamichhane S, Koirala S, Singh SN: Effect of granisetron in attenuation of hypotension following spinal anaesthesia in parturients undergoing elective caesarean section-a double blind randomized controlled trial. JSAN. 2016, 3:13-7. 10.3126/jsan.v3i1.14635

21. Behdad S, Saberi V, Saberi H: Investigating the effect of granisetron on the prevention of hypotension after spinal anesthesia in cesarean section. Journal of Basic and Clinical Medicine. 2016, 5:22-5.

22. Jarisch A, Richter H: Die afferenten Bahnen des Veratrineffektes in den Herznerven (Article in German) . Naunyn Schmiedebergs Arch Exp Pathol Pharmakol. 1939, 193:355-71. 10.1007/BF01859921

23. Sayed AE, Mohamed AS: Ondansetron versus granisetron - effects on hemodynamic instability during spinal anesthesia for caesarean section. Eur J Pharm Med Res. 2017, 4:758-65.

24. Zhang N, He L, Ni JX: Level of sensory block after spinal anesthesia as a predictor of hypotension in parturient. Medicine (Balt). 2017, 96:e7184. 10.1097/MD.0000000000007184

25. Manitshana N: Occurrence and Duration of Spinal-Induced Hypotension in Caesarean Section Patients at an Academic Hospital. Johanesberg; 2016.

26. American Academy of Pediatrics; Committee on Fetus and Newborn; American College of Obstetricians and Gynecologists; Committee on Obstetric Practice: The Apgar score. Adv Neonatal Care. 2006, 6:220-3. 10.1016/j.adnc.2006.04.008 